Color Science and Photometry for Lighting with LEDs and Semiconductor Nanocrystals

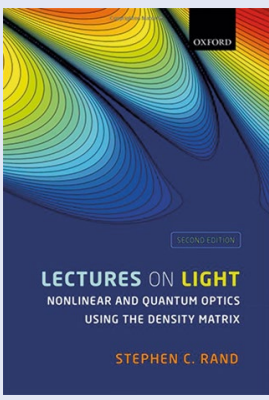

\section{Lectures on Light}

By Stephen C. Rand

OXFORD UNIVERSITY PRESS: 2019. 400PP. $€ 29.99$.

The seven chapters of this book attempt to bridge the gap between introductory quantum mechanics and the research frontier of modern optics and scientific fields that make use of light. Topics discussed include basic classical concepts, basic quantum mechanics, atom-field interactions, coherent transients, coherent interactions of fields and atoms, and quantized fields and coherent states. In this second edition, new sections on quantum interference, Fano resonance, laser cooling of solids, quantum information processing and transverse optical magnetism have been added. The number of references to the topics of ultrafast science, nonlinear optics, $X$-ray and high-field physics have doubled, and there is a new appendix on irreducible representations of magnetic interactions.

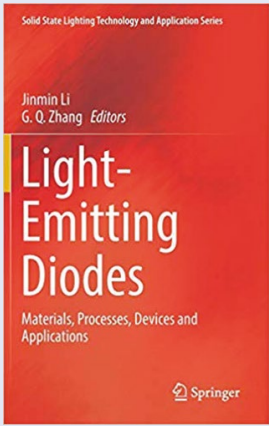

\section{Light-Emitting Diodes}

Edited by Jinmin Li and G. Q. Zhang

SPRINGER: 2019. 600PP. E119.99.

This volume reviews the latest progresses of theories, technologies and applications of lightemitting diodes based on III-V semiconductor materials, such as basic material physics, key device issues, packaging and system integration. The latest developments of light-emitting diodes with working wavelengths ranging from the ultraviolet to the visible are detailed. Material growth, chip structure, packaging and reliability, as well as emerging and novel applications beyond general and conventional lighting, are discussed in the 15 chapters.

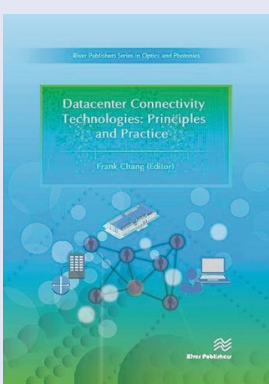

\section{Datacenter Connectivity Technologies: Principles and Practice}

\section{Edited by Frank Chang}

RIVER PUBLISHERS: 2018. 758PP. €85.00.

Optical interconnect technologies at high speed play a critical role for the growth of mega data centres. This book overviews the development of various optical connectivity technologies that are making an impact on the building of data centres. The technologies described span from short-range connectivity, as low as 100 metres with multimode fibre links inside data centres, to long distances of hundreds of kilometres with single-mode fibre links between data centres. Topics discussed are wide ranging. For example, data centre requirements, directly modulated lasers, electro-absorption-modulated lasers, discrete multi-tone modulation, silicon photonics, optical waveguide devices and packaging, and optical coherent networks are covered. 